\title{
Le tourisme dans les Amériques : territoires, expériences et nouveaux enjeux?
}

El turismo en las Américas: territorios, experiencias y nuevos desafíos?

Tourism in the Americas: Territories, Experiences and New Issues?

o turismo nas Américas: territórios, experiências e novos desafios?

\section{Vincent Andreu-Boussut et Elodie Salin}

\section{(2) OpenEdition}

Journals

\section{Édition électronique}

URL : https://journals.openedition.org/ideas/4515

DOI : $10.4000 /$ ideas.4515

ISSN : 1950-5701

\section{Traduction(s) :}

Tourism in the Americas: Territories, Experiences and New Issues? - URL : https://

journals.openedition.org/ideas/4717 [en]

O turismo nas Américas: territórios, experiências e novos desafios? - URL : https://

journals.openedition.org/ideas/5691 [pt]

El turismo en las Américas: territorios, experiencias y ¿nuevos desafíos? - URL : https://

journals.openedition.org/ideas/5721 [es]

Éditeur

Institut des Amériques

Référence électronique

Vincent Andreu-Boussut et Elodie Salin, «Le tourisme dans les Amériques : territoires, expériences et nouveaux enjeux? », IdeAs [En ligne], 12 | 2018, mis en ligne le 10 décembre 2018, consulté le 18 octobre 2022. URL : http://journals.openedition.org/ideas/4515; DOI : https://doi.org/10.4000/ideas. 4515

Ce document a été généré automatiquement le 18 octobre 2022

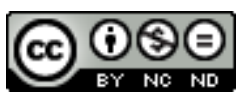

Creative Commons - Attribution - Pas d'Utilisation Commerciale - Pas de Modification 4.0 International - CC BY-NC-ND 4.0

https://creativecommons.org/licenses/by-nc-nd/4.0/ 


\title{
Le tourisme dans les Amériques : territoires, expériences et nouveaux enjeux?
}

\author{
El turismo en las Américas: territorios, experiencias y nuevos desafíos? \\ Tourism in the Americas: Territories, Experiences and New Issues? \\ o turismo nas Américas: territórios, experiências e novos desafios?
}

Vincent Andreu-Boussut et Elodie Salin

Si le continent américain ne constitue que la troisième destination mondiale du tourisme international après l'Europe et la région Asie-Pacifique, le fait touristique n'y demeure pas moins important avec 210,9 millions d'arrivées internationales pour l'année 2017, soit $16 \%$ des arrivées touristiques mondiales (OMT-UNWTO, 2018). Plus important encore, quelques pays y disposent également d'un tourisme intérieur extrêmement développé comme les Etats-Unis ${ }^{1}$ ou le Canada. Ce marché intérieur est d'ailleurs largement prépondérant pour un certain nombre des pays les plus développés et en croissance rapide comme le Brésil. L'ensemble de ces flux touristiques est donc très structurant pour les économies, les sociétés et les territoires des Amériques. A l'échelle de l'ensemble des régions américaines, la mise en tourisme des territoires reste néanmoins un processus inégal et différencié, pour des raisons géohistoriques, culturelles, économiques et politiques. L'Amérique du Nord concentre ainsi la majeure partie des touristes internationaux avec 137 millions d'arrivées touristiques en 2017 tandis que la même année ces visiteurs étaient seulement 36,7 millions en Amérique du Sud, 26 millions dans les Caraïbes et 11,2 millions en Amérique centrale. Dans cette géographie déséquilibrée, les Etats-Unis font figure de leader incontesté sur le plan du tourisme national comme international, se classant régulièrement comme troisième pays le plus visité au monde, comme en 2017 avec 76,9 millions de visiteurs internationaux (OMT-UNWTO, 2018), assez loin devant le Mexique (39,3 millions) et le Canada (20,8 millions). Les destinations les plus importantes du continent n'émergent que très loin derrière avec à peine plus de 6 millions d'arrivées touristiques internationales : l'Argentine, le Brésil, le Chili puis la République dominicaine. Si cette 
situation s'explique en partie par l'ancienneté du développement touristique aux EtatsUnis ou au Canada dès les années 1850, mais aussi en Argentine dès les années 1880, elle traduit aussi et surtout les disparités du niveau de développement économique des pays et reproduit l'hégémonie nord/sud habituellement observable sur les plans économique et socio-politique. Le tourisme a des effets cumulatifs bien connus : les pays les plus riches sont plus touristiques et bénéficient également de ses retombées économiques. Il faut ainsi garder à l'esprit que les Etats-Unis à eux-seuls cumulent les recettes touristiques les plus importantes au monde avec 1035,7 milliards de dollars dépensés en 2017 au titre du tourisme international ${ }^{2}$ et national (US Travel Association, 2018).

2 Cette géographie du tourisme américain se trouve néanmoins remise en cause depuis une bonne vingtaine d'années par le rattrapage des destinations d'Amérique centrale et d'Amérique du Sud qui tend à réduire ces écarts territoriaux. Depuis 1995, le nombre de visiteurs internationaux a ainsi connu une croissance de plus de $330 \%$ pour l'Amérique centrale et de $213 \%$ pour l'Amérique du Sud tandis que cette progression n'a été que de $86 \%$ pour les Caraïbes et de $70 \%$ pour l'Amérique du Nord (OMT-UNWTO, 2018).

Depuis les années 1970, la recherche sur le tourisme s'est largement emparée des espaces américains dans de nombreuses disciplines des sciences sociales (géographie, histoire, anthropologie...) participant à élargir le champ des tourism studies. Les travaux scientifiques nord-américains sur le tourisme ont ainsi pendant longtemps reflété la diversité des dynamiques des territoires touristiques plutôt que d'alimenter des théories du développement du tourisme (Mitchell L., 1984). Développement du tourisme dans les espaces protégés, diversité des formes du tourisme rural au prisme des questions identitaires et foncières, développement urbain par les grands équipements touristiques, continuent à alimenter de nombreuses recherches. Chemin faisant, la littérature scientifique est aussi venue participer de la gestion et du monitoring des territoires en produisant des connaissances ou des outils d'analyse utiles à la décision publique, notamment dans les cas de la gestion urbaine ou des parcs nationaux. Plus largement, les travaux sont aussi nombreux sur l'histoire du tourisme compte tenu du rôle pionnier joué par les Etats-Unis dans l'invention de modèles spatiaux spécifiques (Andreu-Boussut V., 2012), largement diffusés dans le cadre d'une culture de masse du tourisme (Maskintosh W., 2018). Plus récemment, la recherche anglophone comme hispanophone a fait émerger une vision politique des conséquences du phénomène globalisé du tourisme plus spécifiquement en Amérique latine. Cette approche portée en particulier par des ethnologues et des géographes a montré les effets pervers du tourisme assimilé à une extension du colonialisme (Nash D., 1989 ; Mc Cannell D., 1976) ou analysé comme une réalité postcoloniale (Hall M. et H. Tucker, 2004 ; Martinez Mauri M., 2018). Cette dénonciation critique du tourisme a glissé aujourd'hui vers une analyse de la dépendance qui insiste sur les inégalités nord/sud et sur les inégalités de classes dans le cadre de la globalisation (Marie Dit Chirot C., 2018; De Cássia Ariza da Cruz, 2018). Suite aux grandes conférences des Nations-Unies sur le développement durable (Sommet de la Terre de Rio, 1992, Objectifs du Millénaire pour le développement, 2000), la recherche se structure néanmoins désormais en lien avec la gestion publique des territoires, les mouvements sociaux et le tourisme communautaire. Au Brésil et ailleurs en Amérique latine, les études sur le tourisme traitent ainsi régulièrement des articulations entre environnement, espaces protégés et inclusion sociale (Irving M., 2015). 


\section{Le tourisme, facteur de recomposition des territoires : de la consolidation des destinations aux nouveaux fronts touristiques}

4 Le tourisme est avant tout un puissant facteur de transformation des territoires. Dans tout le continent américain, il est à l'origine de la production historique et contemporaine d'aménagements spécialisés qui explique la différenciation et la diversification de toute une typologie d'espaces touristiques. Dans un tel processus, trois dimensions méritent une attention privilégiée: les conditions de la mise en tourisme des territoires, le processus de développement du tourisme sur le temps long et les conséquences territoriales du développement touristique.

$5 \quad$ S'interroger sur les facteurs explicatifs de la mise en tourisme des territoires consiste à expliciter et à déconstruire ce que certains géographes français appellent le moment de lieu (Equipe MIT, 2005). L'émergence de nouveaux lieux sur le planisphère des destinations touristiques s'explique habituellement par l'évolution des représentations culturelles, l'invention et le succès de pratiques de tourisme et par l'évolution du pouvoir d'achat. Au Brésil, Hervé Théry montre ainsi le rôle que joue la croissance de la classe moyenne depuis le gouvernement Lula dans la structuration d'un secteur touristique puissant où le tourisme national est largement prépondérant sur le tourisme international. La consolidation des deux destinations majeures du Nordeste et de la région de Sao Paulo s'explique alors par la proximité de bassins émetteurs de touristes dont les moyens financiers s'améliorent et par la construction d'un « désir » de vacances. Beaucoup plus rarement mobilisé, le changement climatique est aussi à l'origine de la structuration de nouvelles destinations dans des territoires dont le décloisonnement alimente la mise en place de fronts pionniers du tourisme. Pour Alain Grenier, le réchauffement climatique et la fonte des glaces de l'Arctique canadien favorisent depuis une vingtaine d'années l'émergence d'un tourisme de croisière polaire pour lequel l'imaginaire de la frontière inaccessible se voit réactivé pour des touristes fortunés. Le développement de ce tourisme d'aventure participe d'un paradoxe : d'un côté, l'émergence de nouvelles routes maritimes mondiales bouleverse la géopolitique mondiale, et d'un autre côté, l'ouverture économique du territoire inuit renouvelle les besoins de protection des patrimoines et de participation des populations inuites. Au sujet du rôle des représentations dans la mise en tourisme des territoires, Serge Jaumain dissèque la production des guides touristiques imprimés depuis les années 1960 au sujet de la présentation de l'histoire du Québec. Le guide apparaît comme un révélateur de l'évolution des représentations d'un territoire et le reflet de ses transformations socio-culturelles et politiques. Les guides touristiques anglophones et francophones du Québec témoignent ici de la revalorisation progressive des populations autochtones comme de la politisation accentuée de la présentation de l'histoire nationale.

6 Le développement du tourisme sur le temps long peut ensuite être analysé sur le plan géopolitique et économique, car les grandes destinations se transforment généralement au gré d'une concurrence largement mondialisée des marchés touristiques, comme sur le plan des modèles territoriaux à l'œuvre. Le géographe Richard W. Butler a ainsi conceptualisé un modèle de croissance ou de décroissance des destinations selon un cycle de vie, le Tourism Area Life cycle (TALC), dont la célébrité scientifique tient 
beaucoup à son utilisation dans la compréhension du développement de nombreux espaces américains qu'il s'agisse de parcs nationaux, d'espaces urbains ou de stations balnéaires (Butler R., 2006a, 2006b). Michel Desse, Jusline Rodné, Monique Gherardi et Simon Charrier montrent ainsi que le développement touristique des îles des Caraïbes, qui s'appuie notamment sur les croisières (Dehoorne 0., 2007 ; Dehoorne O. et N. Petit-Charles, 2011), s'explique à la fois par la conjoncture économique mondiale et par la manière dont les acteurs publics et privés locaux s'engagent dans le processus de développement. Deux modèles de développement s'y affrontent : un modèle ségrégatif fermé, dont la généalogie est celle de la resort community, et un modèle de développement intégré aux espaces de vie des habitants.

Enfin, les effets du développement touristique sur les territoires et leurs sociétés sont devenus une thématique classique des études sur le tourisme notamment en Amérique latine (Wilson T., 2008). Sur ce sujet, à travers l'analyse fouillée de la mise en tourisme de territoires producteurs de cacao en République dominicaine et au Costa Rica, Laura Henry explore la valorisation de cette filière de production agricole par le tourisme. Bien loin des imaginaires du tourisme balnéaire dominicain et du tourisme de nature costaricain, le développement du tourisme rural sert à la fois de vitrine de la qualité du cacao tout en favorisant l'empowerment des femmes. Plus largement, si les sciences sociales révèlent régulièrement le pouvoir subversif du tourisme pour les sociétés, elles démontrent aussi son pouvoir transformatif, questionnant les dynamiques territoriales sous le prisme de la durabilité.

\section{Des expériences pour repenser l'équilibre entre tourisme, sociétés et environnement : la durabilité en question(s)}

Dans la littérature scientifique et technique, le territoire américain est régulièrement mobilisé pour ses expérimentations de développement durable du tourisme à l'échelle locale, à l'image du guide de bonnes pratiques publié récemment par l'Organisation mondiale du tourisme et l'Organisation des Etats américains (UNWTO et Organization of American States, 2018). Il faut dire que les interrogations sur un meilleur équilibre à trouver entre le développement touristique et la protection des milieux naturels sont nées très tôt aux Etats-Unis. L'invention et la mise en œuvre des premiers parcs nationaux dès les années 1870 aux Etats-Unis puis au Canada s'accompagnent en effet de manière concomitante de leur mise en tourisme (Butler R. et S. Boyd, 2000 ; Depraz S. et S. Héritier, 2012). Dans les années 1930, la croissance rapide d'un public citadin de visiteurs et de campeurs à la recherche d'une reconnexion à la nature, est alors à l'origine d'impacts importants sur les écosystèmes protégés. Terence Young retrace ainsi le rôle pionnier que joue Emilio P. Meinecke dans l'invention des premières mesures de gestion de ces impacts écologiques et dans la formalisation d'un véritable modèle de gestion des flux de visiteurs dans les espaces naturels (zonage des usages, canalisation du public, règles spécifiques d'aménagement des campings...) qui s'est largement exporté depuis hors des Etats-Unis. A Campground Policy publié en 1932 reste d'ailleurs une référence pour l'aménagement des campings accessibles aux automobiles dans les parcs nationaux dont le design imaginé par Meinecke permet à la fois d'abaisser l'empreinte écologique des campeurs et de renforcer leur immersion dans la nature. Depuis lors, bon nombre d'espaces protégés américains sont devenus des hauts lieux 
touristiques pour lesquels cet équilibre entre la protection des milieux naturels et le maintien voire la croissance de la fréquentation reste un enjeu primordial. Le choix des moyens de gestion les plus appropriés y est souvent l'objet d'une gouvernance locale. Dans le Parc national de Guadeloupe, Vincent Andreu-Boussut, Caroline Rufin-Soler et Céline Chadenas montrent que la mise en œuvre de cette gouvernance butte sur des visions divergentes des acteurs socio-économiques locaux concernant le degré d'ouverture au tourisme et le niveau d'intensité des usages, malgré une conscience patrimoniale relativement partagée. Si les espaces protégés terrestres disposent aujourd'hui d'outils éprouvés en matière de gestion du tourisme, le domaine marin fournit un territoire nouveau où les expériences de tourisme durable et de gestion des usages touristiques restent récents (zonage des usages, chartes de bonnes pratiques...).

En dehors des espaces protégés, lieux privilégiés d'expérimentations, le développement balnéaire de masse engagé au Mexique comme dans les Caraïbes dès les années 1970 (Weaver D., 1990 ; Hiernaux D., 2007 ; Dehoorne O. et al., 2010) est très rapidement critiqué pour ses impacts environnementaux et la faiblesse de ses retombées économiques pour les populations locales (Weaver D., 2001 ; Hiernaux-Nicolas D., 2008). Dans un tel contexte, des pratiques touristiques alternatives se développent (Weaver D., 2001) si bien que c'est un architecte mexicain, Héctor Ceballos-Lascurain, qui donne la première définition de l'écotourisme en 1983 (Ballantyne R. et J. Packer, 2013). Le modèle écotouristique, dont les retombées économiques servent à financer les projets de protection des milieux naturels menés par les communautés locales, connaît alors un vif succès (Ceballos-Lascurain H., 1987) dans les îles caribéennes (Douglas J., 1992 ; Weaver D., 1993) et en Amérique latine. Finalement, dans l'ensemble du continent américain, certains acteurs locaux expérimentent des capacités de développement touristique et démontrent ainsi que l'économie du tourisme est loin d'être toujours seulement aux mains de grands groupes économiques nationaux ou internationaux. Au Québec, Sylvain Salaméro, Gilles Caire et Christiane Gagnon montrent que dans les territoires en difficulté démographique et économique, les acteurs socio-économiques du tourisme (communautés autochtones, petites entreprises...) se mobilisent sous la forme de coopératives de solidarité où la gouvernance multipartite est un principe central de gestion des activités touristiques et où la redistribution des retombées économiques alimente la dynamique locale de l'emploi. La vitalité du tourisme coopératif québécois ne doit néanmoins pas occulter la difficulté pour celui-ci à produire des impacts économiques capables d'enrayer les dynamiques régionales de déclin.

Si le changement climatique menace désormais la durabilité des territoires touristiques, il offre aussi un horizon d'opportunités et impose des expérimentations et des innovations. En Guadeloupe, pour Vincent Andreu-Boussut, Caroline Rufin-Soler et Céline Chadenas, c'est la disparition programmée des îlets du Grand Cul-de-Sac Marin qui oblige finalement le Parc national à engager une réflexion collective avec les acteurs économiques sur le futur des usages touristiques. Aux Etats-Unis, Matthew Mc Court et Gabriel Perkins montrent que si le changement climatique menace le tourisme hivernal, il est également une formidable opportunité pour souder la société civile sur un projet de skiable village de plus grande durabilité territoriale. A Bethel, dans le Maine, une communauté de volontaires a ainsi pris en main l'organisation d'une compétition de ski nordique pour éviter son annulation pour des raisons climatiques. En renommant l'événement sportif « Protect our Winters », ils lui ont attribué un objectif 
d'atténuation du changement climatique en limitant les déplacements automobiles des participants le temps de la compétition. Si les émissions évitées de $\mathrm{CO} 2$ restent faibles au regard des émissions totales produites par les participants à l'échelle de leurs déplacements touristiques, les bénéfices de cette expérimentation et du travail bénévole restent nombreux pour le territoire, qu'il s'agisse du développement local induit par les dépenses des visiteurs ou de la reconnaissance des capacités de la société locale à construire un projet partagé de développement touristique pertinent pour répondre à des enjeux d'environnement global.

\section{L'importance de la question communautaire dans le développement du tourisme}

11 Le tourisme communautaire, s'il est perçu comme un moteur de développement économique et social, correspond à une pensée qui s'est développée à la fin des années 1990 qualifiée de «tourisme pro-pauvre» (Figueroa Pinedo J. et al., 2014). Générant ainsi accessibilité, opportunités, emplois dans des régions enclavées et défavorisées, le tourisme communautaire peut également être le déclencheur de prise de conscience pour la diversité culturelle et la sauvegarde puis la valorisation des patrimoines qu'ils soient naturels, culturels ou immatériels. Les populations autochtones du Yucatán contribuent en effet au développement local de leur communauté en restaurant leur patrimoine naturel et en participant à la mise en oeuvre d'un programme national de tourisme alternatif en zone indigène. En déconstruisant une initiative de tourisme communautaire, Samuel Jouault souligne l'émergence de lieux pionniers qui proposent une alternative aux pratiques touristiques dans une région marquée par le tourisme de masse (Riviera Maya). La mise en tourisme de l'arrière-pays maya, à travers l'exemple d'un village yokdzonot, illustre la transformation et l'inversion des hiérarchies spatiale et sociale. Si le cenote (grotte inondée formant un puit à ciel ouvert) a longtemps été perçu comme un lieu marginalisé jusqu'à l'utiliser comme décharge à ciel ouvert, il est désormais transformé en un lieu central, aménagé pour la plongée, et géré de manière communautaire.

12 Malgré de nombreux exemples vertueux, des critiques d'un tourisme pro-pauvres s'élèvent (Harrison D., 2008) dénonçant l'incapacité du tourisme à réduire la pauvreté ou à résoudre des problèmes sociaux et environnementaux dans les communautés locales. L'exemple des indiens Gunas au Panama montre que, bien qu'autogéré, le tourisme autochtone n'est pas nécessairement durable. L'entretien réalisé par Johanna Durget et Elodie Salin avec Iniquipili Chiari témoigne d'une facette en demi-teinte du tourisme communautaire dans la région littorale autonome de Guna Yala. Le modèle touristique, exclusivement contrôlé par les Gunas, propose des activités balnéaires se déployant sur les nombreuses îles de l'archipel. Loin de l'écotourisme, ce modèle de gestion communautaire très hiérarchisé semble favoriser une approche non durable de l'activité touristique très rémunératrice et génératrice de conflits sociaux et environnementaux, tant dans la société Guna qu'avec le gouvernement panaméen.

13 Alors, en quoi l'écotourisme peut-il être une opportunité pour les population autochtones et un choix de développement pour une destination touristique ? Dans la forêt amazonienne du Brésil, Bastien Beaufort s'intéresse au projet écotouristique Vinte Quilos mis en place par les indiens Sateré Mawé autour d'une ressource agricole et patrimoniale, le guarana, et centré sur le concept d'ethno-développement. Cette 
opportunité de développement d'un tourisme communautaire s'appuie sur un projet de commerce équitable mis en place depuis 1994. Si les résultats d'une mise en tourisme sont encore balbutiants, les perspectives d'une mise en tourisme partielle et timide de l'Amazonie brésilienne semblent néanmoins faire émerger un possible front pionnier touristique. Dans l'émergence de ces nouvelles destinations, Alain Grenier pose clairement la question de la participation des populations locales car dans l'Arctique canadien la mise en tourisme des territoires inuit se fait en grande partie sans les communautés.

\section{Mises en tourisme et patrimonialisations : une co- construction?} de leur énonciation, orientant un renouvellement des approches, avec l'apparition de nouvelles catégories comme celle des paysages culturels en 1992. Elodie Salin propose une analyse comparée de trois espaces labellisés paysages culturels de l'Unesco à travers l'argumentaire de leurs valeurs universelles exceptionnelles. Qu'il s'agisse du café en Colombie, de l'Agave (plante de la Tequila) au Mexique ou de la Quebrada de Humahuaca en Argentine, les dossiers de candidatures des trois paysages culturels tentent de construire un discours, une narration patrimoniale, au service de la mise en tourisme des territoires. L'importance des images et des discours, parfois extérieurs et/ ou portés par des acteurs spécifiques des territoires, est ici mise en exergue afin de montrer l'émergence d'une identité, voire d'une marque territoriale forte, passant par une esthétisation des paysages, quitte à construire une image factice, conforme à l'image que les visiteurs se font des lieux. Ces nouveaux récits opèrent un changement de signification du bien par un passage du local au mondial (Djament-Tran G. , E. Fagnoni, S. Jacquot, 2012).

16 La fabrique patrimoniale en tant que construction politique, sociale et culturelle s'inscrit dans un contexte de globalisation où les attentes des communautés locales peuvent entrer en conflit avec celles des décideurs. Les conflits de gestion des sites et les enjeux de l'appropriation, y compris sur le plan immatériel et symbolique, soulèvent des enjeux de gouvernance patrimoniale et touristique. Le patrimoine peut alors être intégré aux politiques touristiques comme un avantage comparatif qui renforce l'attractivité des territoires. Elsa Broclain, Linda Boukhris, Sébastien Jacquot et Elodie Salin soulignent le rayonnement international et l'importance de la pratique du tango dans la ville de Buenos Aires, nourrissant un imaginaire fécond et un marketing territorial au service du réancrage du tango dans son berceau originel. La démonstration de l'instrumentalisation du tango, déclaré patrimoine culturel immatériel en 2009 par les autorités de Buenos Aires, s'accompagne de multiples 
initiatives privées qui créent une véritable économie touristique du tango. La tension est néanmoins forte entre un patrimoine immatériel globalisé et donné à voir aux touristes dans les tango-shows ou les méga-événements et un tango plus social identifié par les acteurs portègnes du monde tanguero comme patrimoine local. L'idée d'une résistance à une forme hégémonique de patrimonialisation, accompagnant la mise en tourisme des lieux, est reprise par Sébastien Jacquot qui propose une étude de la ville de Valparaiso au prisme de l'émergence controversée d'une destination du tourisme culturel. Pour inverser l'image d'une ville en crise, la municipalité mise sur un tourisme patrimonial renforcé par l'inscription au patrimoine mondial en 2003. Les logiques de protection et de labellisation, accompagnées de grands projets urbanistiques, engendrent des mutations urbaines, sociales et entrepreneuriales qui transforment les paysages urbains. Les controverses portées par les habitants visent à remettre en question les processus de gentrification, d'éviction des populations locales résidentes, de spéculation et de touristification excessives des cerros patrimonialisés aboutissant à une transformation des usages et des appropriations des quartiers Unesco. Une dilatation des pratiques touristiques est également observable au-delà des zones les plus emblématiques, produisant une géographie touristique alternative et patrimoniale de la ville.

\section{BIBLIOGRAPHIE}

Andreu-Boussut, Vincent, «Les espaces du tourisme en Amérique du Nord », in Cynthia GhorraGobin C. et Alain Musset (dir.), L’Amérique du Nord, Paris, Ed. Armand Colin, 2012, p. 83-102.

Ballantyne, Roy, et Packer, Jan (éd.), International Handbook on Ecotourism, Edward Elgar Publishing, 2013.

Bourdeau, Laurent, Gravari-Barbas, Maria, et Robinson, Mike (dir.), Tourisme et patrimoine mondial, Québec, Presses de l'Université Laval, 2012.

Boyd, Stephen W., « The TALC Model and its Application to National Parks : A Canadian Example », in Richard W. Butler (éd.), The Tourism Area Life Cycle vol. 1, Applications and Modifications, Clevedon, Channel View Publications, Aspects of Tourism, 2006, p. 119-138.

Butler R. W. et Boyd, Stephen W., " Tourism and Parks. A long but uneasy relationship », in Richard W. Butler et Stephen W. Boyd (éd.), Tourism and National Parks. Issues and Implications, Chichester, Ed. Wiley, 2000, p. 3-11.

Butler, Richard W. (éd.), The Tourism Area Life Cycle vol. 1, Applications and Modifications, Clevedon, Channel View Publications, 2006a.

Butler, Richard W. (éd.), The Tourism Area Life Cycle vol. 2. Conceptual and Theoretical Issues, Clevedon, Channel View Publications, 2006b.

Ceballos-Lascurain, Hector, « The Future of"Ecotourism” », Mexico Journal, 1987, p. 13-14.

De Cássia Ariza da Cruz, Rita, « Desenvolvimento desigual e turismo no Brasil », Confins, 36, 2018, http://journals.openedition.org/confins/13707, page consultée le 19 octobre 2018. 
Dehoorne, Olivier, « La Baie du Marin (Martinique) : l'organisation d'un nouvel espace touristique autour de la plaisance ", Études caribéennes, vol. 7, 2007, https://journals.openedition.org/ etudescaribeennes/381, page consultée le 21 octobre 2018.

Dehoorne, Olivier, Murat, Christelle, et Petit-Charles, Nathalie, « International tourism in the Caribbean Area : Current Status and Future Prospects », Études caribéennes, vol. 16, 2010, https:// journals.openedition.org/etudescaribeennes/4713, page consultée le 21 octobre 2018.

Dehoorne, Olivier, et Petit-Charles, Nathalie, « Tourisme de croisière et industrie de la croisière », Études caribéennes, vol. 18, 2011, http://etudescaribeennes.revues.org/5623, page consultée le 20 octobre 2018.

Depraz, Samuel, et Héritier, Stéphane, « La nature et les parcs naturels en Amérique du Nord », L'Information géographique, $\mathrm{n}^{\circ}$ 4, vol. 76, 2012, p. 6-28.

Douglas, Joy E., « Ecotourism : The future for the Caribbean ? ", Industry and Environment, vol. 15, 1992, p. 64-66.

Djament-Tran, Géraldine, Fagnoni, Edith, et Jacquot, Sébastien, « La construction de la valeur universelle exceptionnelle dans la valorisation des sites du patrimoine mondial, entre local et mondial », in Laurent Bourdeau, Maria Gravari-Barbas et Mike Robinson (dir.), Tourisme et patrimoine mondial, Québec, Presses de l'Université Laval, 2012, p. 217-234.

Equipe MIT, Tourismes 2. Moments de lieux, Paris, Ed. Belin, 2005.

Fagnoni, Edith, Les espaces du tourisme et des loisirs, Paris, Ed. Armand Colin, 2017.

Figueroa Pinedo, Jessica, Arellano, Alexandra, Tello-Rozas, Sonia « Développement touristique ou reproduction sociale de la pauvreté ", Téoros, 33, 2, 2014, http://journals.openedition.org/teoros/ 2679, page consultée le 09 novembre 2018.

Gravari Barbas, Maria, Jacquot, Sébastien, Patrimoine mondial et développement, : au défi du tourisme durable, Presses de l'Université du Québec, 2014.

Hall Collin Michael, Tucker Hazel (eds.), Tourism and postcolonialism : Contested discourses, identities and representations, Londres, Routledge, 2004.

Harrison, David, « Pro-poor Tourism : A Critique », Third World Quarterly, vol. 29, no 5, 2008, p. 851-868.

Hiernaux, Daniel, « Le tourisme de masse au Mexique : un virage ? », Téoros, vol. 26, $\mathrm{n}^{\circ}$ 1, 2007, p. $15-20$.

Hiernaux-Nicolas, Daniel, « Mexico : Tensions in the Fordist Model of Tourism Development », in Lily M. Hoffman, Susan S. Fainstein et Dennis R. Judd (éd.), Cities and Visitors. Regulating People, Markets and City Space, Wiley-Blackwell Publishing, 2008, p. 187-199.

Irving, Marta de Azevedo, Rodrigues, Camila Gonçalves de Oliveira, Rabinovici, Andrea, Costa, Helena Araújo (Orgs.), Turismo, áreas protegidas e inclusão social : diálogos entre saberes e fazeres, Rio de Janeiro, Ed. Folio Digital, Letra e Imagem, 2015.

Mackintosh, Will B., Selling the Sights : The Invention of the Tourist in American Culture, NYC, New York University Press, 2018.

Marie dit Chirot, Clément, « Vers une économie politique du tourisme. Réflexion à partir de quelques expériences latino-américaines », Norois, n 247, 2018, p. 7-13, https://www-cairninfo.doc-elec.univ-lemans.fr/revue-norois-2018-2-page-7.htm, page consultée le 9 novembre 2018. 
Martínez Mauri, Mònica, « ¿Por qué pagar por entrar a Gunayala ? Movilidad turística, soberanía y pueblos indígenas en Panamá », Norois, n²47, 2018, p. 63-76, https://www-cairn-info.docelec.univ-lemans.fr/revue-norois-2018-2-page-63.htm, page consultée le 9 novembre 2018. Mc Cannell Dean, The tourist. A new theory of the leisure class, New York, Shocken Books, 1976.

Mitchell, Lisle S., « Tourism research in the United States : a geographic perspective », Geojournal, vol. $9, \mathrm{n}^{\circ} 1,1984$, p. 5-15.

Nash, Dennison, « El turismo considerado como una forma de imperialismo », in Smith (éd.), Anitriones e invitados. Antropología del Turismo, Madrid, Endymion, 1992, p. 69-95.

OMT-UNWTO, Faits saillants OMT du tourisme, Edition 2018, Madrid, OMT-UNWTO, 2018,. UNWTO et Organization of American States, Tourism and the Sustainable Development Goals, Good Practices in the Americas, Madrid, UNWTO, 2018.

US Travel Association, 2018, US Travel and Tourism Overview (2017), Research Fact Sheet, NYC.. Weaver, David B., « Grand Cayman Island and the resort cycle concept », The Journal of Travel Research, vol. 29, n², 1990, p. 9-15.

Weaver, David B., « Ecotourism in the small island Caribbean », GeoJournal, vol. 31, n 4, 1993, p. 457-465.

Weaver, David, " Mass Tourism and and Alternative Tourism in the Caribbean ", in David Harrison (éd.), Tourism and the less developed world : Issues and case studies, Wallingford, CAB International Publishing, 2001, p. 161-174.

Wilson, Tamar D., « Introduction : The Impacts of Tourism in Latin America », Latin American Perspectives, vol. 35, $\mathrm{n}^{\circ}$ 3, 2008, p. 1-20.

\section{NOTES}

1. Les Etats-Unis ont comptabilisé 2,25 milliards de départs individuels en séjour touristique (visites-personnes) en 2017 (US Travel Association, 2018).

2. Sur le plan du seul tourisme international, les Etats-Unis se partagent $18 \%$ des parts du marché touristique mondial.

\section{AUTEURS}

\section{VINCENT ANDREU-BOUSSUT}

Maître de conférences en géographie. UMR ESO 6590 CNRS, Le Mans Université. vincent.andreuboussut@univ-lemans.fr 


\section{ELODIE SALIN}

Maîtresse de Conférences en géographie, Le Mans Université, Laboratoire Espaces et Sociétés (UMR ESO 6590 CNRS), Chercheuse associée EIREST (Equipe interdisciplinaire de Recherche Sur le Tourisme) - Université Paris I Panthéon-Sorbonne 